\title{
NOUVELLE TECHNIQUE DE DÉTERMINATION DU COEFFICIENT D'EFFICACITÉ PROTÉIQUE (C. E. P.) ET SA JUSTIFICATION ${ }^{1}$
}

\author{
J. ABRAHAM et J. PERETIANU \\ avec la collaboration technique de M. A. DE Saintaurin \\ Centre de Recherches sur la Nutrition, C. N. R. S., Bellevue (Seine-et-Oise).
}

En donnant séparément à l'animal un apport azoté contrôlé en quantité et en le laissant libre de consommer les calories, on arrive à allouer toujours la même quantité d'azote tout en se plaçant dans les conditions optimales d'équilibre entre protides et énergie, conditions qu'on ne peut pas réaliser avec des régimes mixtes. L'usage de deux repas séparés, une ration azotée contrôlée en quantité et un régime calorique alloué ad libitum, semble donc se prêter favorablement à l'établissement du Coefficient d'Efficacité Protéique (gains de poids par gramme de protides ingéré) en utilisant le rat comme réactif animal.

La nouvelle technique de détermination du coefficient d'efficacité protéique que nous proposons est basée sur ce fait.

Dans un tel type d'expérience, il convient de conditionner les animaux à ingérer leur repas protéique dans un temps donné : 1 heure. Il s'agit de rations isoazotées réalisées en " diluant" avec de l'amidon les protides les plus concentrés et en ajoutant ensuite systématiquement $10 \%$ de sucre pour limiter l'influence de l'appétence. Ce mélange est distribué à sec et procure à chaque animal $180 \mathrm{mg}$ d'azote par jour. Il est capital que cet apport soit entièrement consommé. Le régime calorique à base de sucre est également alloué sous forme sèche. Les animaux en disposent à volonté 23 heures sur 24. Pratiquement, il est utile d'étudier les animaux durant une prépériode de 8 jours. Pendant celle-ci tous les rats sont nourris comme nous l'avons indiqué précédemment, mais la ration protéique de "démarrage " est constituée d'un mélange en parties égales des protéines à tester. On peut ainsi éliminer les animaux qui sont inaptes au conditionnement.

L'expérience proprement dite dure 28 jours au cours desquels on détermine le gain de poids en fonction des protides ingérés (C. E. P.). Les résultats peuvent être exprimés en valeur absolue ou relative (en donnant la valeur 100 à la protéine de référence).

1. Le texte détaillé de ce mémoire est en cours d'impression aux Annales de la Nutrition et de l'Alimentation. Nous ne donnons ici que l'essentiel de la technique et les derniers développements dont elle a fait l'objet. 


\section{MÉTHODE}

Sur la base de ces observations préliminaires, voici le protocole expérimental que nous avons utilisé :

Quatre protéines sont à tester : caséine + cystine, farine de poisson, tourteaux de soja et d'arachide. Le régime énergétique contient $85 \%$ de saccharose, sans autre source de glucides.

Une cinquantaine de rats mâles de souche Wistar C. F. pesant en moyenne $40 \mathrm{~g}$ sont observés durant la prépériode de 8 jours. On retient les animaux ayant consommé au moins $90 \%$ du repas protéique. Au cours de l'expérience proprement dite ( 28 jours) les ingérés sont déterminés quotidiennement et les animaux pesés tous les 4 jours. Dans ce cas précis, nous avons calculé conjoin. tement l'ingéré protéique et l'ingéré calorique pour mettre en évidence le meilleur paramètre à utiliser pour classer les protides testés.

\section{RÉSULTATS}

Ils ne portent que sur les animaux ayant consommé au moins $95 \%$ de l'apport protéique. Le tableau 1 rapporte les résultats d'ensemble. Cette expérience montre que la vitesse de croissance est fonction de la qualité des protéines et que le taux protéique réalisé spontanément par l'animal est en rapport inverse avec celle-ci.

\section{Comparaison entre les paramètres utilisés.}

Pour obtenir une première indication sur l'intérêt pratique des paramètres utilisés dans le tableau 1 pour la classification relative des protides étudiés, nous rapportons dans le tableau 2 leurs valeurs relatives en prenant comme référence la caséine.

Les chiffres se rapportant au gain de poids et au C. E. P. semblent permettre une différenciation plus précise que ceux se rapportant à l'ingéré calorique et au rapport " calories ingérées/azote ingéré ". Il en résulte donc que le paramètre le plus intéressant est le C. E. P., qui présente l'avantage supplémentaire de ne pas requérir la détermination de l'ingéré calorique.

\section{DISCUSSION}

On pourrait faire a priori un certain nombre d'objections à la méthode que nous avons proposée; aussi avons-nous tenu à en éluder quelques-unes.

\section{A. Influence de la nature de l'apport énergétique.}

\section{$1^{\circ}$ Glucides.}

Dans une de nos expériences nous avons été amenés à remplacer dans le régime énergétique le saccharose par l'amidon. Cette substitution n'a eu aucun effet ni sur la croissance des animaux, ni sur leur consommation. Par contre nous avons constaté l'absence totale des cas de porphyrine que nous avions remarqués avec le régime au saccharose.

Signalons que ces cas de porphyrine n'étaient pas dus à une mauvaise couverture du besoin en vitamines du groupe B; une surcharge de ces métabolites sous forme purifiée ou sous forme de levure, tant dans la ration énergétique que dans la ration protéique, n'avait été ni préventive ni curative. 
DÉTERMINATION DU COEFFICIENT D'EFFICACITÉ PROTÉIQUE

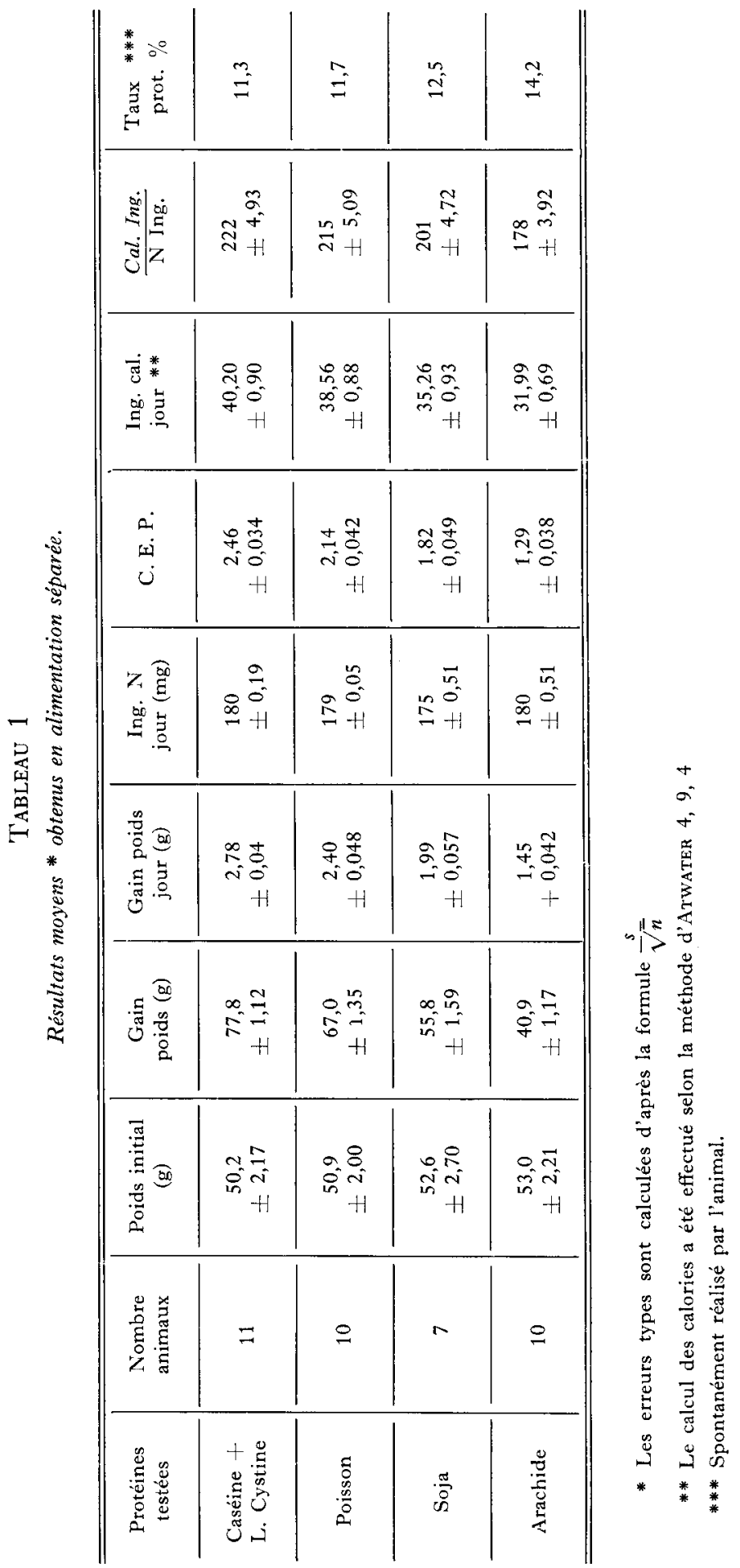




\section{TABleaU 2}

Comparaison entre les différents paramètres utilisés par rapport à la caséine.

\begin{tabular}{c|c|c|c|c}
\hline \hline $\begin{array}{c}\text { Protéines } \\
\text { Paramètres }\end{array}$ & Caséine & Poisson & Soja & Arachide \\
\hline $\begin{array}{c}\text { Gain de poids } \\
\text { journalier }\end{array}$ & 100 & 86,3 & 71,5 & 52,1 \\
\hline $\begin{array}{c}\text { C. E. P. } \\
\text { journalier }\end{array}$ & 100 & 86,9 & 73,9 \\
\hline$\frac{100}{\text { Calories ingérées }}$
\end{tabular}

\section{$2^{\circ}$ Lipides.}

On peut se demander si l'animal consomme une quantité déterminée d'énergie (exprimée en calories) pour couvrir un besoin métabolique (donc fonction de la qualité et de la quantité de l'ingéré protéique) où s'il ingère une quantité globale de nourriture liée à sa capacité digestive. Nous avons donc modifié la teneur en calories de la ration énergétique en y incorporant un taux variable de lipides, en l'occurrence du saindoux, afin de voir les répercussions de cette modification sur l'efficacité protidique de la ration. Nous avons utilisé cinq taux de lipides : $5,15,20,25$, et $35 \%$ et une protéine : la farine de poisson.

Voici les résultats de l'expérience qui a duré 28 jours ('Tableau 3).

\section{TABleau 3}

Influence du taux de lipides dans le régime énergétique en alimentation séparée (résultats moyens).

\begin{tabular}{c|c|c|c|c}
\hline $\begin{array}{c}\text { Lot } \\
\text { p. 100 de lipides }\end{array}$ & $\begin{array}{c}\Delta \mathbf{P} \\
\mathbf{g}\end{array}$ & $\begin{array}{c}\text { N ingéré } \\
\text { mg }\end{array}$ & Calories & C. E. P. \\
\hline & & 4665 & 1001 & 1,61 \\
5 & 47 & 4650 & 966 & 1,58 \\
15 & 46 & 4600 & 993 & 1,72 \\
20 & 49 & 470 & 963 & 1,89 \\
35 & 55 & 4720 & 972 & 1,55 \\
\hline
\end{tabular}


On constate que le phénomène d'ajustement se vérifie pleinement. Les animaux ont mangé la totalité de leur ration protéique et si on observe une légère augmentation dans les ingérés de calories avec le régime à $25 \%$, cette différence n'est pas significative.

De ces quelques résultats préliminaires on peut conclure avec prudence que le rapport " calories/protéines " spontanément établi par le rat dans nos conditions expérimentales est probablement de nature métabolique et non pas fonctionnelle.

\section{B. Influence de l'état physiologique.}

Nous avons voulu étendre aux adultes les observations faites sur l'animal en croissance.

\section{Entretien.}

Nous avons suivi quelques animaux mâles et femelles adultes auxquels nous avons fourni $180 \mathrm{mg}$ d'azote par jour et par animal, sous forme de soja pour un lot et d'arachide pour l'autre; les résultats sont rapportés dans le Tableau 4.

\section{Tableau 4}

Résultats moyens obtenus en alimentation séparée.

Animaux adultes.

\begin{tabular}{c|c|c|c}
\hline \hline Protéine & Sexe & DP total $(\mathrm{g})$ & $\begin{array}{c}\text { Calories totales } \\
\text { ingérées par jour }\end{array}$ \\
\hline \multirow{3}{*}{ Arachide } & Mâles & 10 & 37 \\
& Femelles & 0 & 31 \\
\multirow{2}{*}{ Soja } & Mâles & 49 & 43 \\
& Femelles & 17 & 44 \\
\hline \hline
\end{tabular}

Il existe donc pour l'adulte comme pour le jeune en croissance un rapport « calories-protéines "fonction de la qualité des protéines; ce rapport est supérieur à celui obtenu chez les jeunes. Pour une protéine donnée, la quantité de calories ingérée est sensiblement la même pour les mâles et les femelles, mais leur efficacité est plus grande chez les mâles dont le gain de poids est nettement supérieur à celui des femelles.

\section{$2^{\circ}$ Gestation.}

Après une période d'adaptation au régime, une partie des femelles a été accouplée et nous avons suivi l'évolution du rapport $\mathrm{C} / \mathrm{P}$ pendant leur période de gestation.

Nous avons constaté que la consommation spontanée d'énergie demeurait fonction de la qualité des protéines et que l'état de gestation augmentait l'ingestion de calories. 


\section{Comparaison avec la méthode classique de détermination du C.E.P.}

Il était indispensable de confronter nos résultats avec ceux obtenus par la méthode classique, utilisant un régime mixte donné ad libitum avec taux protéique fixe. Nous avons choisi pour cela trois des protéines utilisées précédemment : caséine + cystine, farine de poisson, tourteau de soja.

Il n'est pas nécessaire de donner les résultats de cette expérience en détail, les quelques chiffres que voici les résumeront suffisamment (Tableau 5).

TABleau 5

Comparaison entre les deux modes d'alimentation.

\begin{tabular}{|c|c|c|c|c|}
\hline & \multicolumn{2}{|c|}{ Alimentation séparée } & \multicolumn{2}{|c|}{$\begin{array}{l}\text { Alimentation mixte } \\
(14 \mathrm{~g} \text { protéines p. } 100)\end{array}$} \\
\hline & $\Delta \mathrm{P} /$ jour $(\mathrm{g})$ & C. E. P. & $\Delta \mathrm{P} /$ jour $(\mathrm{g})$ & C. E. P. \\
\hline Caséine + cystine & $2,93 \pm 0,10$ & $2,69 \pm 0,08$ & $4,11 \pm 0,26$ & $2,24 \pm 0,08$ \\
\hline Farine de poisson & $2,48 \pm 0,07$ & $2,21 \pm 0,07$ & $4,47 \pm 0,22$ & $1,93 \pm 0,05$ \\
\hline Tourteau de soja & $2,11 \pm 0,06$ & $1,99 \pm 0,04$ & $3,77 \pm 0,25$ & $1,69 \pm 0,07$ \\
\hline
\end{tabular}

On voit, tout d'abord, que la hiérarchie obtenue à l'aide des gains de poids bruts diffère selon le type d'alimentation. En valeur absolue, le poisson $(4,47 \mathrm{~g} / \mathrm{j})$ dépasse la caséine $(4,11 \mathrm{~g} / \mathrm{j})$ avec l'alimentation mixte, alors que l'efficacité réelle, mesurée par le C. E. P., est plus élevée pour la caséine que pour le poisson. Cette disparité entre croissance absolue et relative ne se manifeste plus avec l'alimentation séparée : ici la hiérarchie est la même avec les gains de poids bruts et avec le C. E. P.

Pour le reste, il est difficile de procéder à des comparaisons valables entre les deux modes d'alimentation, puisqu'ils diffèrent entre eux par le taux des ingérés caloriques et azotés et par là-même par la vitesse de croissance qu'ils déterminent.

Mais si on se limite à la comparaison des C. E. P. dans l'un et l'autre type d'expérience, on constate que l'alimentation séparée donne une meilleure différenciation entre les protides, du fait de la plus grande homogénéité des résultats. On peu donc assurer statistiquement la signification d'une différence plus petite. La technique proposée est ainsi justifiée. Elle est plus sensible que la méthode classique et permet une meilleure différenciation de la valeur biologique des protides. De même elle est techniquement facile à conduire et demande peu de manipulations. 